permanently attached to instruments without the necessity of removing them from their often delicately adjusted supports.

The instrument was built by Mr. Kalmbach, the laboratory mechanician, to whom I am indebted for some valuable mechanical suggestions.

Randal Morgan Laboratory of Physics

University of Pennsylvania

Philadelphia

March, 1917

\title{
A NEW TYPE OF COMPARISON PHOTOMETER
}

By P. G. Nutting

The Martens comparison photometer* has for years been the favorite instrument for precision work in measuring relative transmission, reflection and relative brightness in general. It gives a good comparison field free from any perceptible dividing line. It gives the highest precision of which a visual instrument is capable and is so compact and well protected that it is seldom injured or thrown out of adjustment.

This is a polarization instrument, however, and if the light enters it in a partly polarized condition, either serious errors result or a tiresome duplication of readings is necessary to eliminate such errors. A much more serious objection, however, is that the instrument is no longer on the market, since the calcite for making the Wollaston and nicol prisms used for varying the light intensity in a known manner is not available. The new instrument was designed to do the precision work for which the Martens photometer is ordinarily used, but with more flexibility to take care of a wider variety of work. The use of calcite and of the polarization principle is avoided in its construction.

In the new type of instrument, the light beam is divided and recombined by means of bi-prisms and the density is controlled in a known manner by means of a precision iris diaphragm or neutral gray wedge inserted at the precise apex of the field pencils. The field viewed is bi-lateral and circular. The scale is empirical and very nearly linear. The two instruments constructed for the Research Laboratory of the Eastman Kodak Company have shown high sensibility and precision.

The construction of the instrument is as shown in the figure. The instrument is symmetrical about a longitudinal plane. In each beam a

\footnotetext{
${ }^{*}$ Phys. Zs. Vol. I, page 299, 1900.
} 
lens, L, throws an image of the field on the apex of the bi-prism, B, of which the angles are equal to those of the wedges, $P$, used to deflect the beams. The field is viewed by means of the ocular, $\mathrm{O}$, focused on the apex of the bi-prism. The lenses used are of short focus and symmetrical, light intensities are varied by means of iris diaphragms, D, which are very simply and carefully made. These diaphragms are carefully located at the intersection of object and image zone pencils. Hence, in viewing the image of the field at $B$, opening and closing the diaphragms varies the intensity of illumination over the whole field uniformly. It is upon this fundamental principle that the operation of the photometer depends, and practical tests of the completed instrument show that the condition is so well fulfilled that no departure from uniformity is perceptible to the eye.

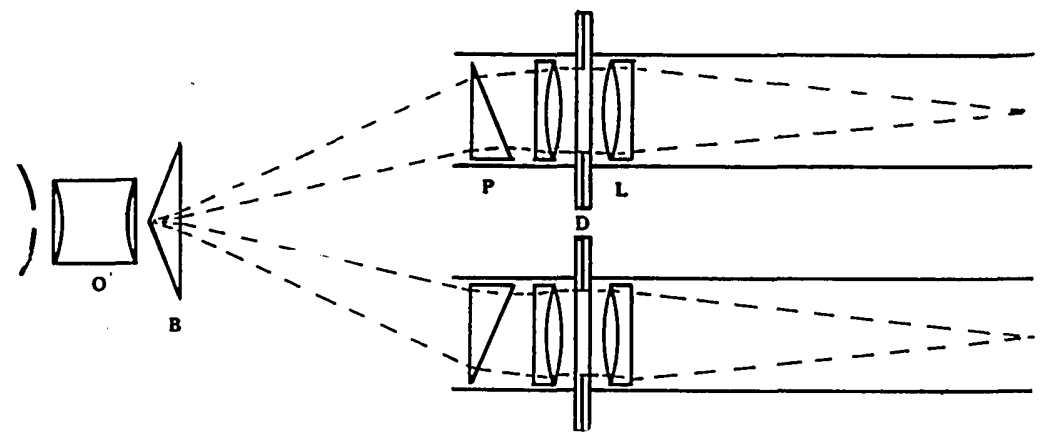

For certain purposes the diaphragms are replaced by neutral tint absorbing wedges about six inches long and half an inch wide enclosed in glass plates, with a range of density from o to about 3.0. Such wedges may be obtained non-selective to within a very small error and with densities very nearly a linear function of the distance along the wedge. Wedge control is preferable for certain classes of work.

To increase the range of relative brightness measurable with this instrument, decimal gray filters about two centimeters square are inserted just in front of either of the two lenses in a brass box provided with suitable ways and light tight. The densities supplied are o (dummy), I, 2, 3, 4 and 5. The corresponding transmissions are I.0, 0. I0, 0.0010, 0.00010, 0.000010 and o.oooooro. These filters are a commercial article and may be had with precision guaranteed to $1 / 2 \%$ of the whole transmission.

The dimensions of the instrument may be varied through wide limits by using lenses of different focal lengths and prisms of various angles. 
The first model constructed was 16 inches long and the second 9 inches over all. The tubes on which the lenses were mounted are about two centimeters in diameter and lined with non-reflecting black felt paper.

As a brightness comparator, the instrument is used in its simplest form and is adapted to measure percentage transmission of thin objects such as photographic negatives. By stopping down the field apertures, the densities of small details may be readily measured. For such work, the illuminated field used as an accessory must be of extreme uniformity. Such a field we have found best obtained by placing a sheet of solid opal glass over the top of a white lined box containing an electric lamp. White drawing paper makes a good lining. The sheet of opal glass should be of pot opal rather than flashed opal, should be fine ground on both surfaces and of uniform thickness. If no lamp is available which does not give streaky illumination, a second plate of rough ground clear glass is placed an inch or two below the opal glass. This arrangement gives an exceedingly uniform illumination.

By means of various accessories attachable to the field ends of the photometer tubes, the instrument may be adapted for use as an illuminometer, brightness photometer, reflectometer, etc.

For use as a brightness photometer, a small light box is slipped over the end of the left hand tube, this box being white lined and containing a small, well seasoned lamp operated on storage battery. For determining the brightness of a distant object, a pair of lenses forming a simple Galilean telescope, such as half of a field glass, is placed axially in front of the right hand photometer tube so that an image of the distant object is thrown in the field plane of the photometer.

For use as an illuminometer, a white screen is mounted at a suitable angle about six inches in front of the right hand photometer tube, and an image of this thrown in the field plane of the instrument by means of a small auxiliary lens.

For use as a reflectometer, pairs of suitable reflecting or refracting prisms are placed over the field aperture of each photometer tube.

The chief advantage of this form of brightness comparator over the Martens is its freedom from errors due to polarization in the light to be measured. Like a Martens, its limit of sensibility is the limit of photometric sensibility of the human retina. Its scale is not quite as convenient as the circular scale, but the range of contrast to which the instrument is 
applicable is very much greater. The instrument is simple in construction and comparatively inexpensive.

I am greatly indebted to various members of the staff of the physics division of the Research Laboratory of the Eastman Kodak Company for expert calibration of the scales and testing of sensibilities of these photometers.

Pittsburg

June, 1917

\section{A COMPENSATING FILTER IN SPECTROSCOPY ${ }^{1}$}

By M. B. Hodgson and R: B. Wilsey

It would be desirable in many investigations in spectroscopy to have a photographic emulsion which would yield, in the same exposure, equal densities in the developed negative, over a wide spectral region, when using a light source having a continuous spectrum. While present day panchromatic dry plates show sensitiveness throughout the whole visible spectrum, and while the modern high efficiency illuminant is constant in radiant output and more uniform in spectral distribution than heretofore, such an ideal condition does not exist; the sensitiveness of the plate is not a constant value for all wave lengths nor is the energy distribution of the illuminant even approximately uniform.

To overcome these inherent defects, in obtaining quantitative or reliable qualitative analyses of absorption bands, various expedients are employed. One such consists in compensating for the lack of uniformity by using colored filters of selective absorption. With these (neglecting the further objection of the work involved in their preparation) it is difficult to get even approximate correction. A second method is to measure optically the density of the silver deposits in negatives exposed both through the medium whose transmission is being studied and without it. By proper computation and a knowledge of the sensitometric constants of the particular plate used, a measure may be had of the true absorption of the medium. This method, however, is open to many objections.

Recently Luckiesh ${ }^{2}$ described a method which he has used with success in obtaining uniform photographic intensity throughout the spectrum, using a light source yielding a continuous spectrum.

${ }^{1}$ Communication No. 42 from the Research Laboratory of the Eastman Kodak Company.

2 Astrophysical Journal, May 1916, p. 302. 